\title{
THE EFFECT OF INTERMITTENT CORTISONE ADMINISTRATION IN THE RABBIT
}

\author{
Induction of Cycles of Resorption and Deposition of Bone \\ Elsdon Storey, Melbourne, Australia \\ From the Department of Pathology, University of Melbourne
}

It was found during studies on the movement of teeth with orthodontic appliances (Smith and Storey 1952, Storey 1953) that variations in the rate of tooth movement, related to the menstrual cycle, occurred in girls (Storey 1954). It was postulated that this cyclic variation in rate of tooth movement was, in part at least, related to cyclic changes in hormonal secretion affecting bone and connective tissues. For this reason study was made of the effect of producing cycles of bone changes by the use of intermittent administration of cortisone.

Previous work had shown that administration of cortisone to the rabbit is followed by rapid resorption of bone (Storey 1957a); and, after its withdrawal, an equally rapid deposition of bone occurs, not only in the resorbed areas but on periosteal surfaces and within sutures (Storey 1957b). The new bone is separated from the old by a darkly staining haematoxylin " reversal" line which, on the periosteal surface of the skull, resembles a layer of bone formed by appositional growth. It is known that periosteal activity during normal (Weinmann and Sicher 1955) or neoplastic growth (Sissons 1949) is also intermittent, as shown by successive rings of bone-particularly on the long bones and the vault of the skull. However, whether these superimposed layers of bone are local in origin or are local manifestations of general hormonal control is not known.

Jaffe, Bodansky and Blair (1932), by injecting rabbits with various doses of parathormone in an attempt to prolong its effect without killing the animals, produced cyclic changes in bone structure. They demonstrated a small series of " reversal "lines in the bone surrounding vessels in the skull; but they made no mention of any periosteal activity.

If normal growth processes in bone are influenced by the cyclic changes of endocrine activity it should be possible, in any attempt to study bone pathology, to induce cycles of bone change that are abnormal in intensity and duration as well as those that are normal. For instance Jaffe (1933) ascribed the change in osteitis deformans to an increased cycle of resorption and deposition of bone which gives rise to the typical mosaic pattern shown in this condition. Selye (1932) also produced bone changes of a different character by administering small doses of parathormone every two days: paradoxically, instead of bone resorption, an increased bone deposition took place.

This present paper is concerned with a preliminary study of cycles of resorption and deposition of bone in the rabbit after intermittent cortisone administration. The results have been used as a basis for a large-scale, long-range experiment now in progress.

\section{EXPERIMENTAL METHOD}

Cortisone acetate was administered to rabbits-eight young (three to five months) and one old (three years) -in doses of twenty-five milligrams a day for various periods; it was then withdrawn for a further period. A period of administration followed by withdrawal of the hormone will be referred to as one cycle. Series of one, two, three and four cycles of cortisone administration were produced in a number of rabbits. The time intervals of the various cycles are shown in Table I. 
Coronal sections of the skull were cut an eighth of an inch behind the coronal suture; specimens were also obtained from the femur and premaxillae. They were decalcified in

TABLE I

Time Intervals of Cortisone Administration

( + refers to administration of cortisone)

( - refers to withdrawal of cortisone)

\begin{tabular}{cccc|}
\hline Cycle & $\begin{array}{c}\text { Cortisone administration } \\
\text { (in days) }\end{array}$ & $\begin{array}{c}\text { Number } \\
\text { of animals }\end{array}$ \\
\cline { 1 - 2 } 2 & $\begin{array}{c}\text { A } \begin{array}{c}+7-2 \\
+7-7\end{array} \\
2\end{array}$ & $+7-7,+7-7$ & 1 \\
3 & $+7-14,+7-7,+7-7$ & 1 \\
4 & $+7-7,+7-7,+7-14,+5-3$ & 2 \\
\hline
\end{tabular}

5 per cent nitric acid; and histological sections were cut at $10 \mu$ and stained with Ehrlich's acid haematoxylin and eosin.

\section{RESULTS}

Normal bone-The bones of normal male and female rabbits of ages corresponding to those in the experiment were examined. In the skull " reversal " or " arrest" lines are found both on the outer and inner plates of the cranial bones, where they run a relatively straight course parallel to the periosteal surface. They vary considerably in numbers in the young animals whereas in the older animal there are more. These lines are not present in the bone adjoining the sagittal or premaxillary sutures; a few are found on the outside of the middle of the shaft of the femur.

INTERMITTENT ADMINISTRATION OF CORTISONE

One cycle $(+7-2$ and $+7-7)-$ The administration of cortisone for seven days followed by two days' withdrawal is associated with resorption of bone of the skull, but the usually observed increases of vascularity and appearance of multinucleated osteoclasts are absent. After seven days' withdrawal of cortisone a general change from resorptive activity to laying down of new bone is most obvious (Storey 1957b).

A new layer of bone has formed on the outer and inner periosteal surfaces of the skull (Fig. 1). This new layer is not continuous along the entire surface of the bone.

Within the sagittal suture there is a thickening of the blue-staining haematoxylin layer at the junction of the sutural connective tissue and bone; in places this widens to form a new thin layer of bone.

New bone also forms on the periosteal surface and within the trabeculae of the premaxilla. There is little change in the growing epiphysial cartilage of the femur; normal growth has not yet been resumed. There are numerous areas within the bone where new bone formation is occurring - a darkly staining " reversal" line separates the old and new bone.

Two cycles $(+7-7 ;+7-7)-$ Two new layers of bone have formed on the outer and inner periosteal surfaces of the skull (Fig. 2), but in places a single layer has formed on the endosteal surface. These layers of bone are separated by two lines which sometimes converge to form one or even disappear; within the sagittal suture two lines are present. Similarly two layers of bone are seen on the periosteal surface of the premaxilla and femur. Within the bone of the skull it is possible to see where two waves of resorption and deposition have formed two " reversal" lines around some small vascular spaces. 


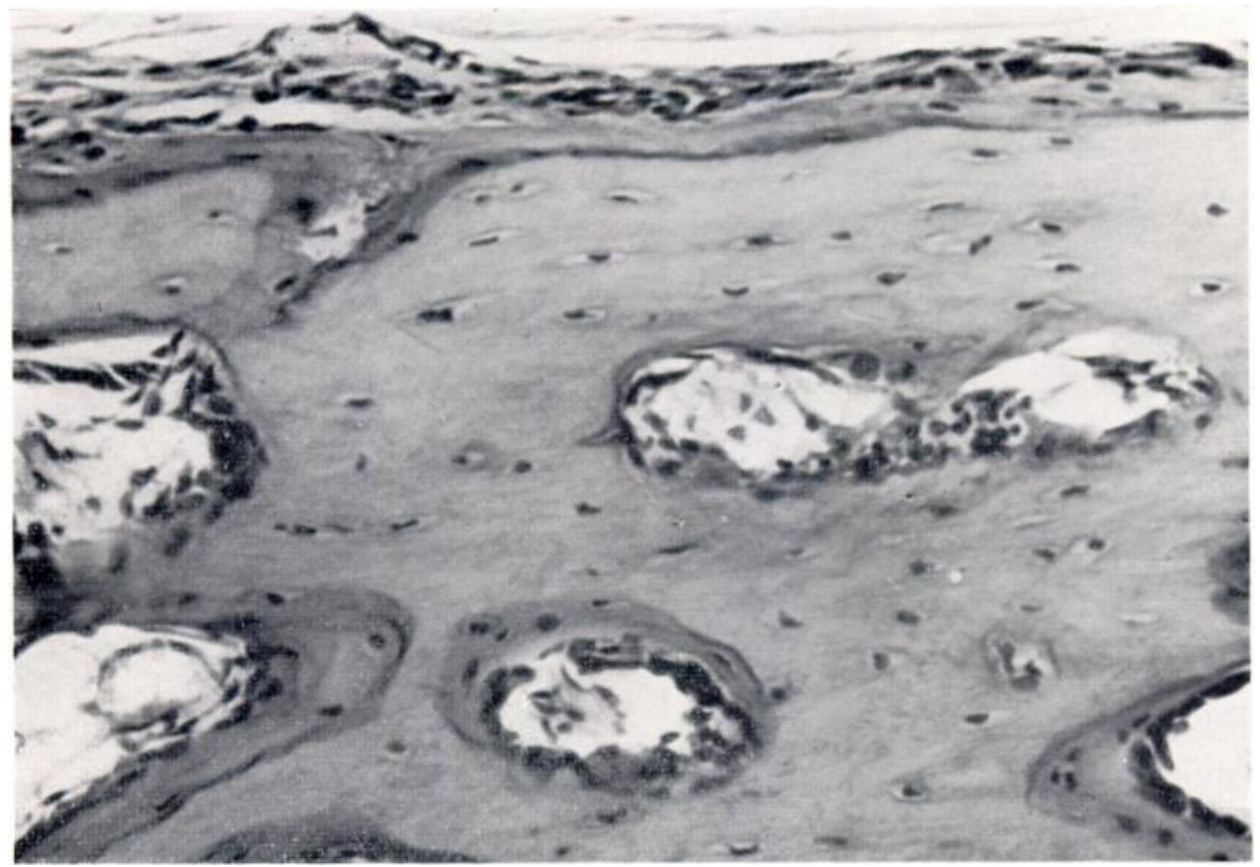

Fig. 1

The effect of one cycle of cortisone administration on the outer plate of bone of the cranial vault in the young rabbit. A new layer of bone is formed on the periosteal surface and around vascular spaces in the bone. This layer of bone is separated from the old bone by a darkly staining haematoxylin line. $(\wedge 400$.

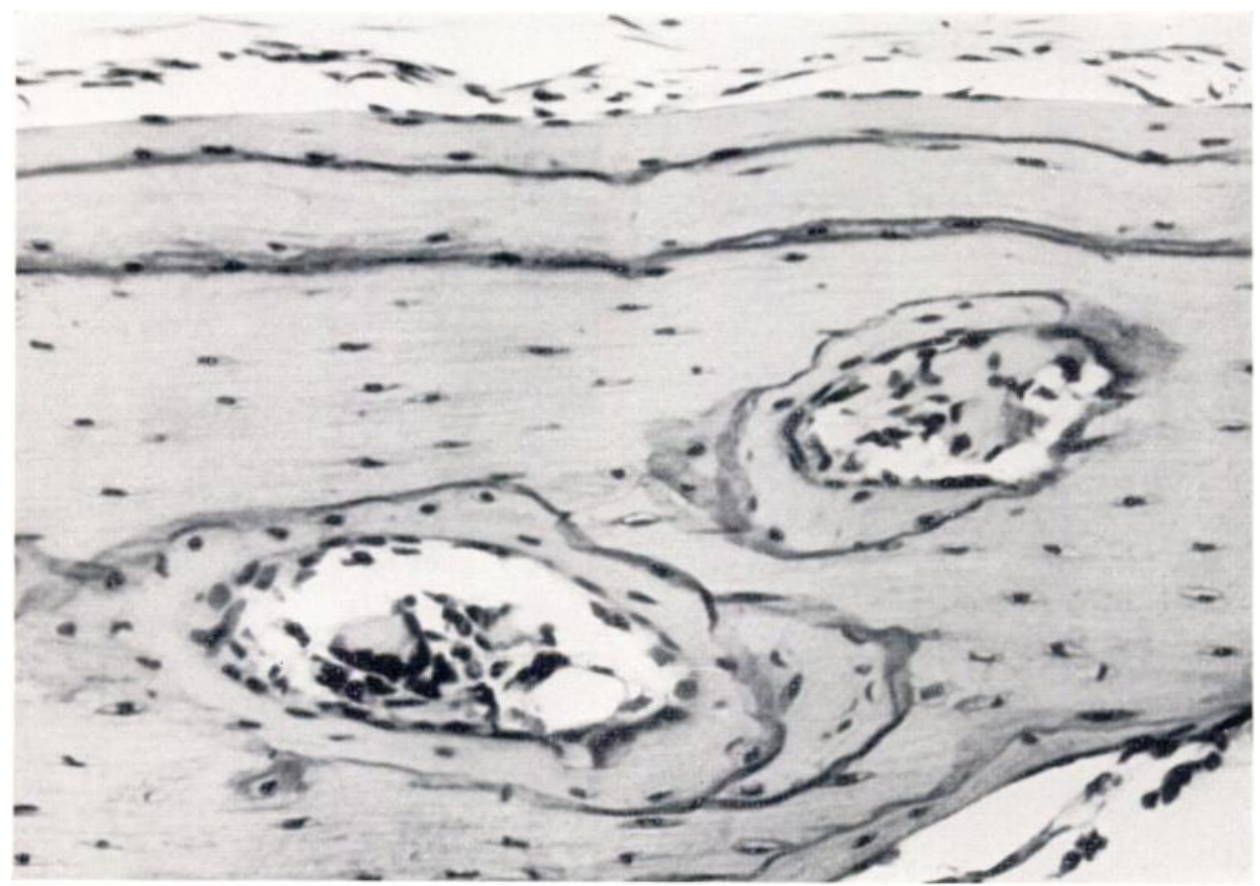

Fig. 2

The effect of two cycles of cortisone administration on the outer plate of bone of the cranial vault in the young rabbit. Two new layers of bone are formed on the periosteal surface separated by two reversal lines. The first line appears double in places. $(\times 400$.) 


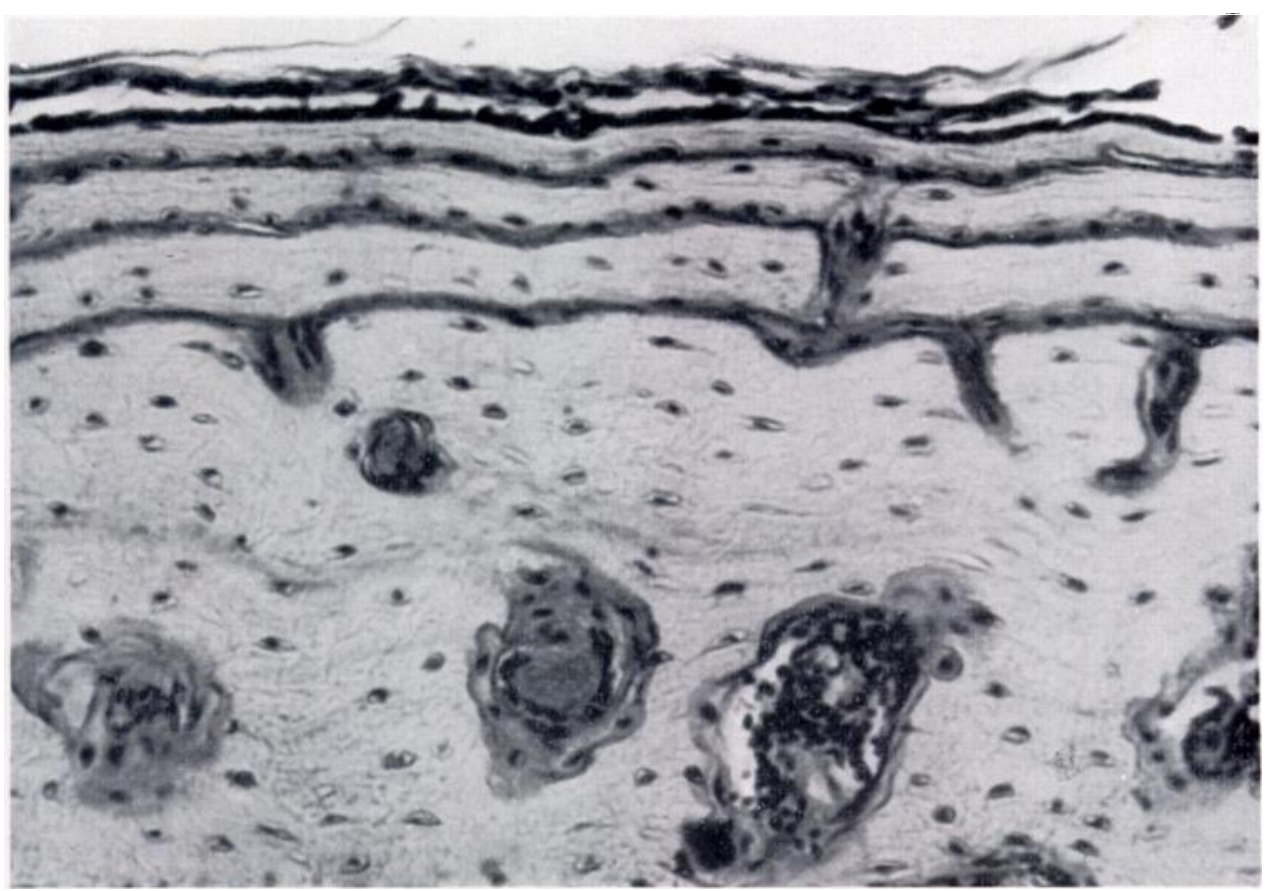

Fig. 3

The effect of three cycles of cortisone administration on the outer plate of bone of the cranial vault in the young rabbit. Three new layers of bone are formed on the periosteal surface. The first layer formed during fourteen days' withdrawal of cortisone is approximately twice as thick as the outer two. $(\times 400$. $)$

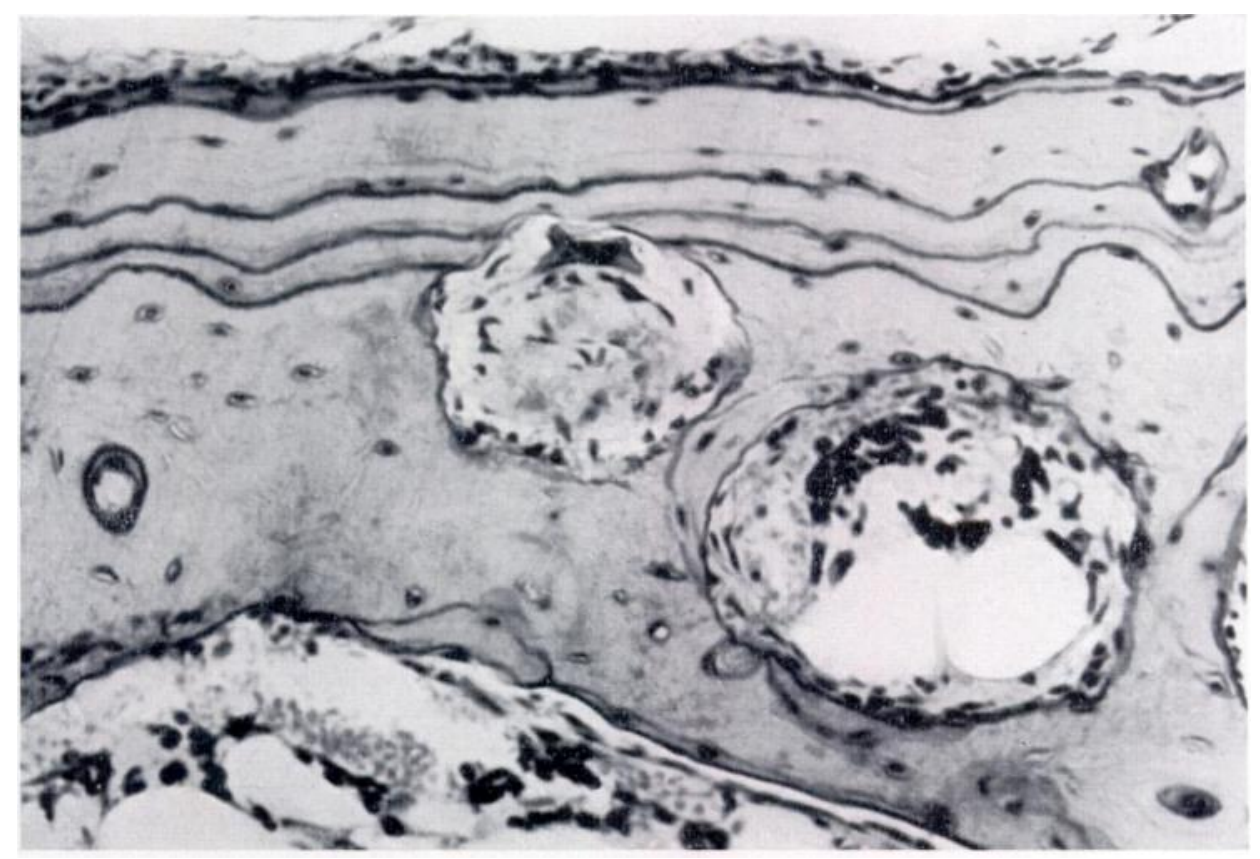

Fig. 4

The effect of four cycles of cortisone administration on the outer plate of bone of the cranial vault in the young rabbit. Four new layers of bone formed on the periosteal surface. The third layer formed during fourteen days' withdrawal of cortisone is twice as thick as the first two; the outer layer is very much narrower, showing the small amount of bone formed during three days' withdrawal of cortisone. The vascular spaces in the bone show areas of resorption in this particular section. $(\times 400$.) 


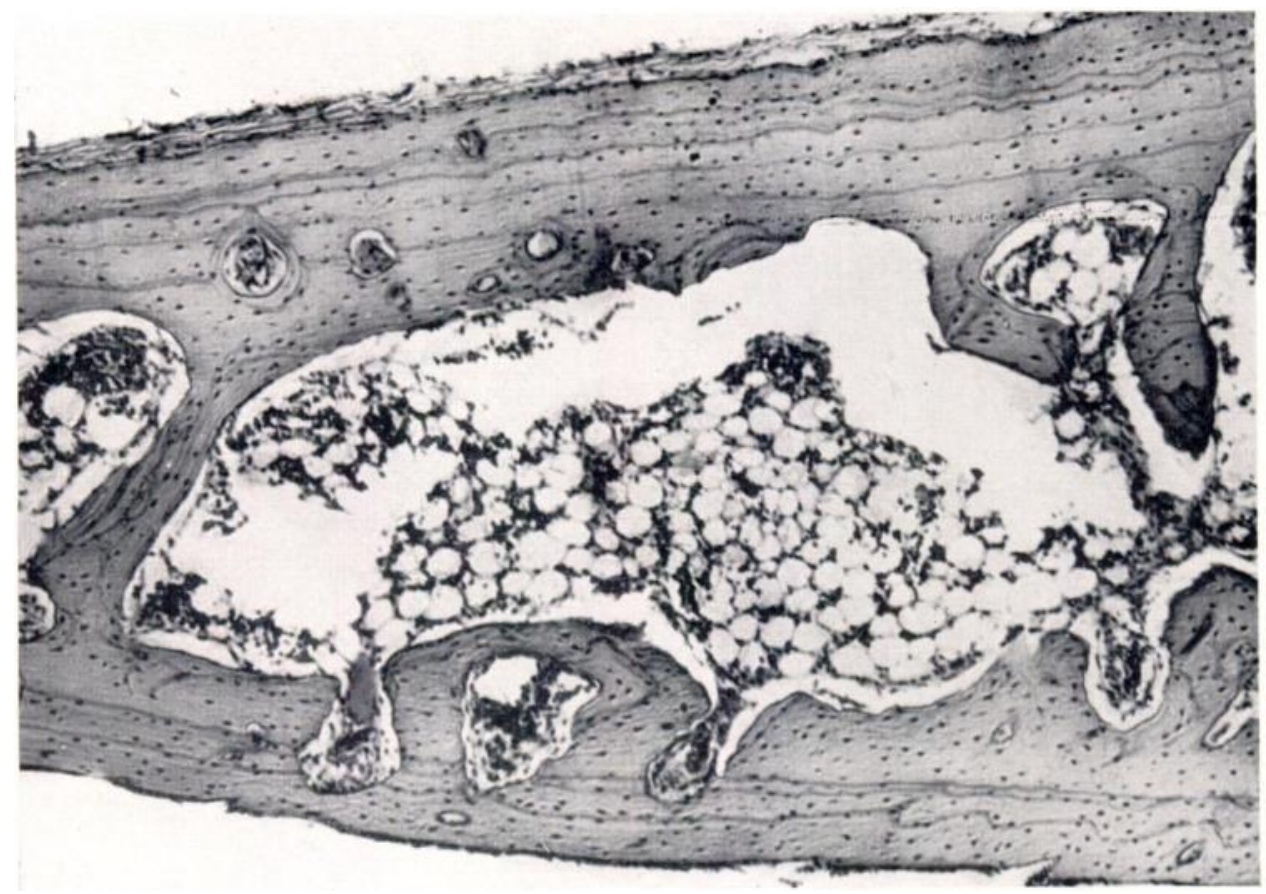

FIG. 5

Coronal section of cranial vault showing the formation of four new layers of bone on both external and internal surfaces after four cycles of cortisone administration in the young rabbit. The fourth layer of new bone has been formed only partly in this section but is shown more clearly in Figures 4 and 7 . $(\div 80$.

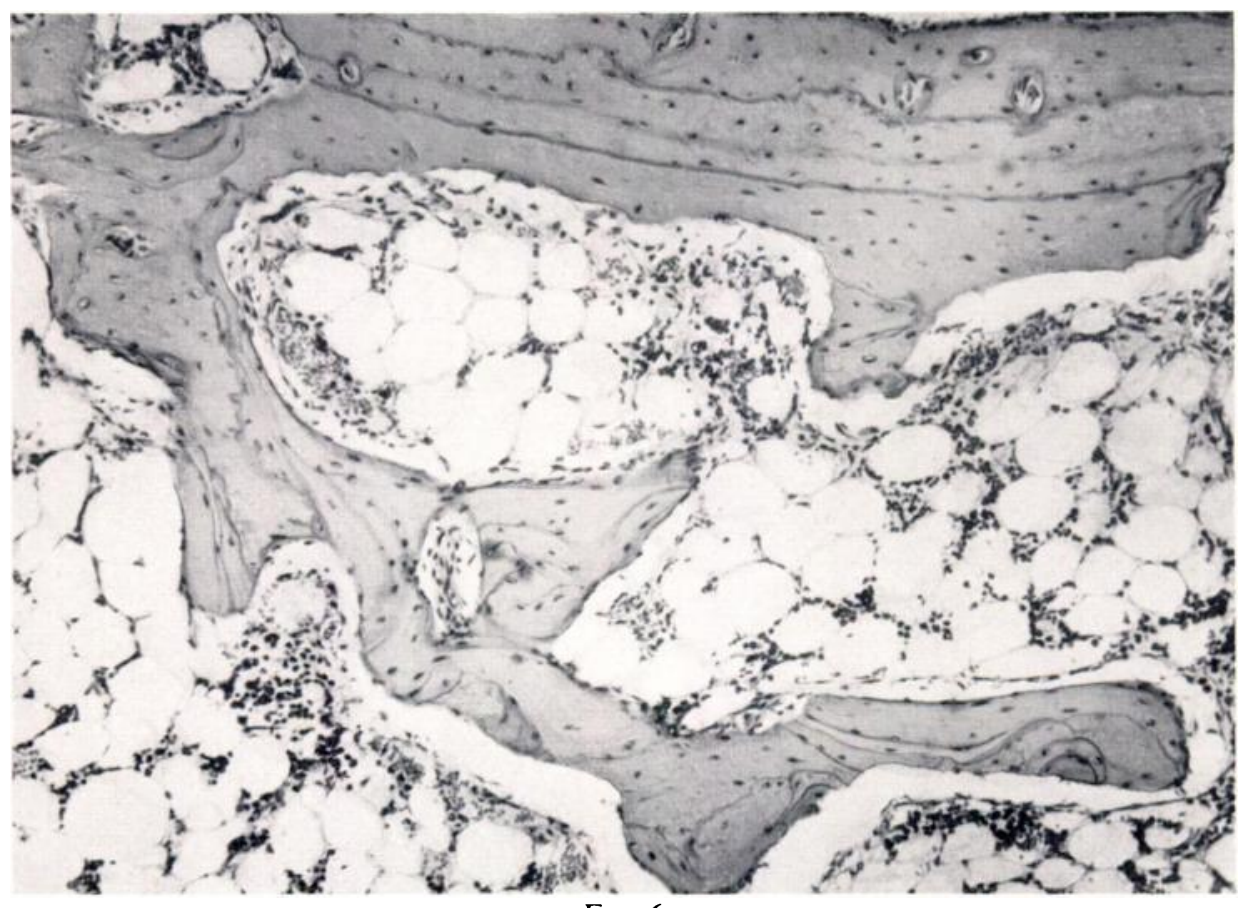

Fig. 6

Area adjacent to the section shown in Figure 5. There are four cycles of cortisone administration. Associated with the new layers of appositional bone growth there are changes in the internal architecture of the trabeculae, which are irregularly arranged and contain numerous "reversal" lines. The fourth layer of new bone is clearly apparent only at the upper right-hand corner. $(\times 240$. $)$ 


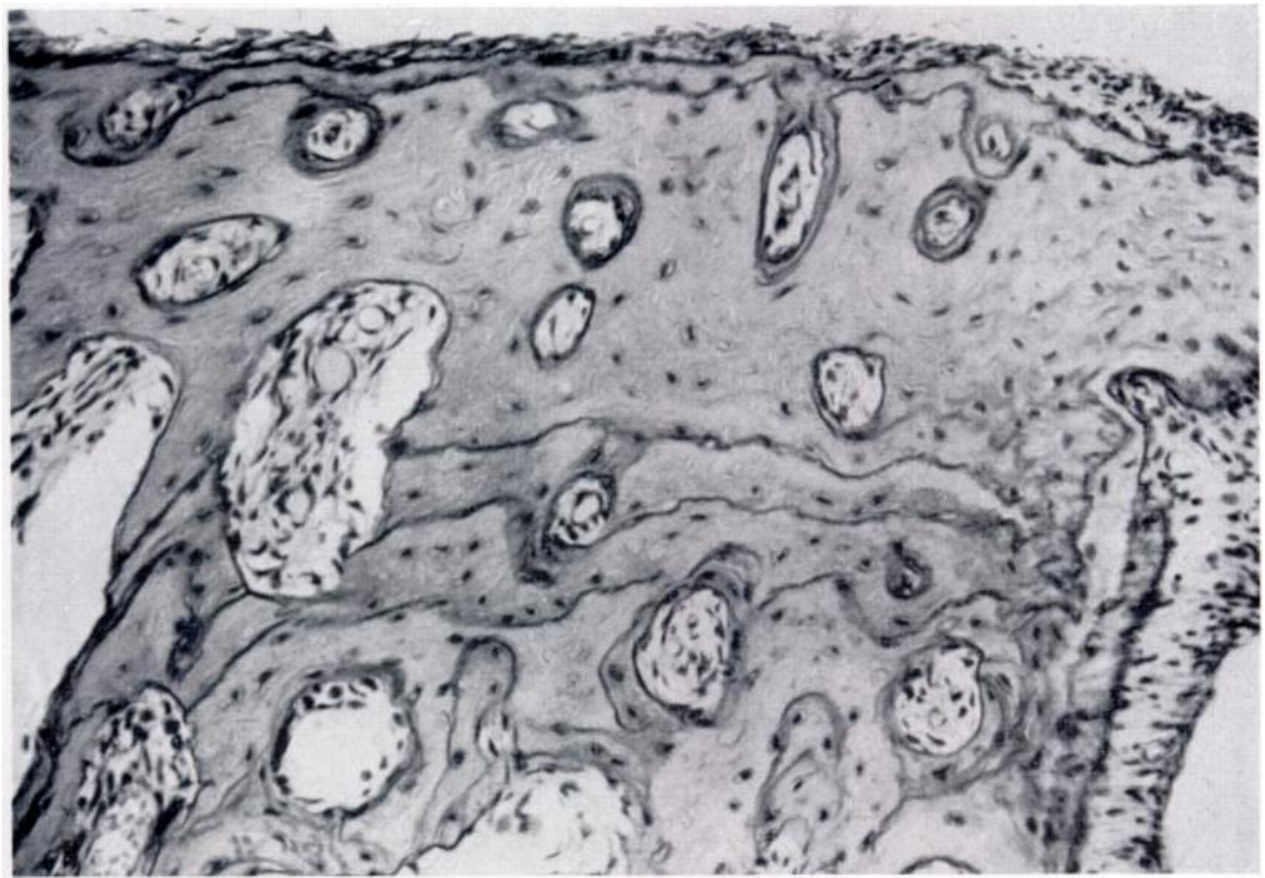

Fig. 7

The effect of four cycles of cortisone administration on the anterior aspect of the cranial vault in the young rabbit. The width of new bone is greater than that formed towards the posterior aspect of the skull in the same animal, although the same sequence of layers is present (compare with Fig. 4). Note the accelerated reconstruction of the underlying bone and the appearance of irregular reversal lines. $(\times 240$.)

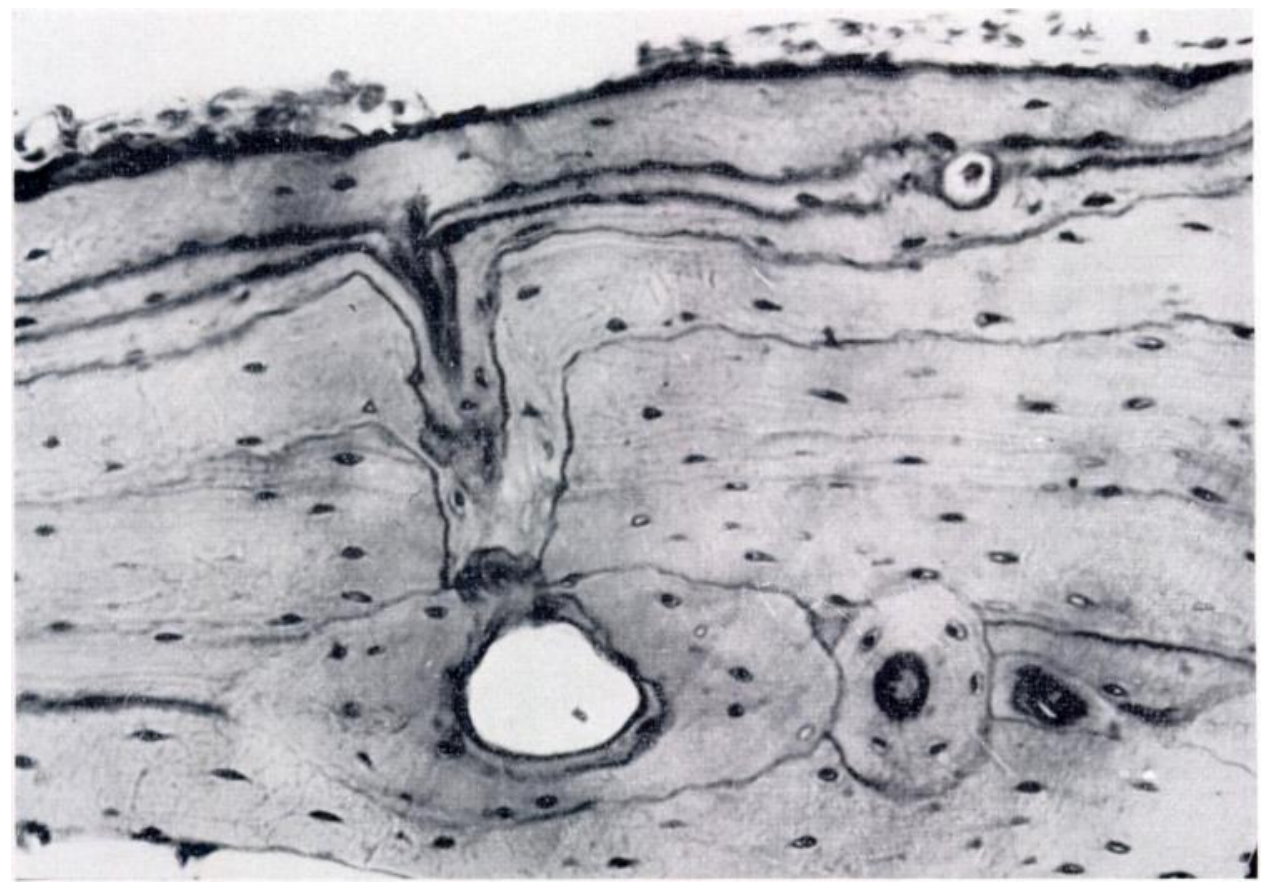

Fig. 8

The effect of four cycles of cortisone administration in the old animal. The width of new bone formed is less than in the young animal although the same sequence of layers is seen. The fourth layer is only just detectable towards the upper left part of the section (compare with Figs. 4 and 7$)$. $(\times 400$.) 


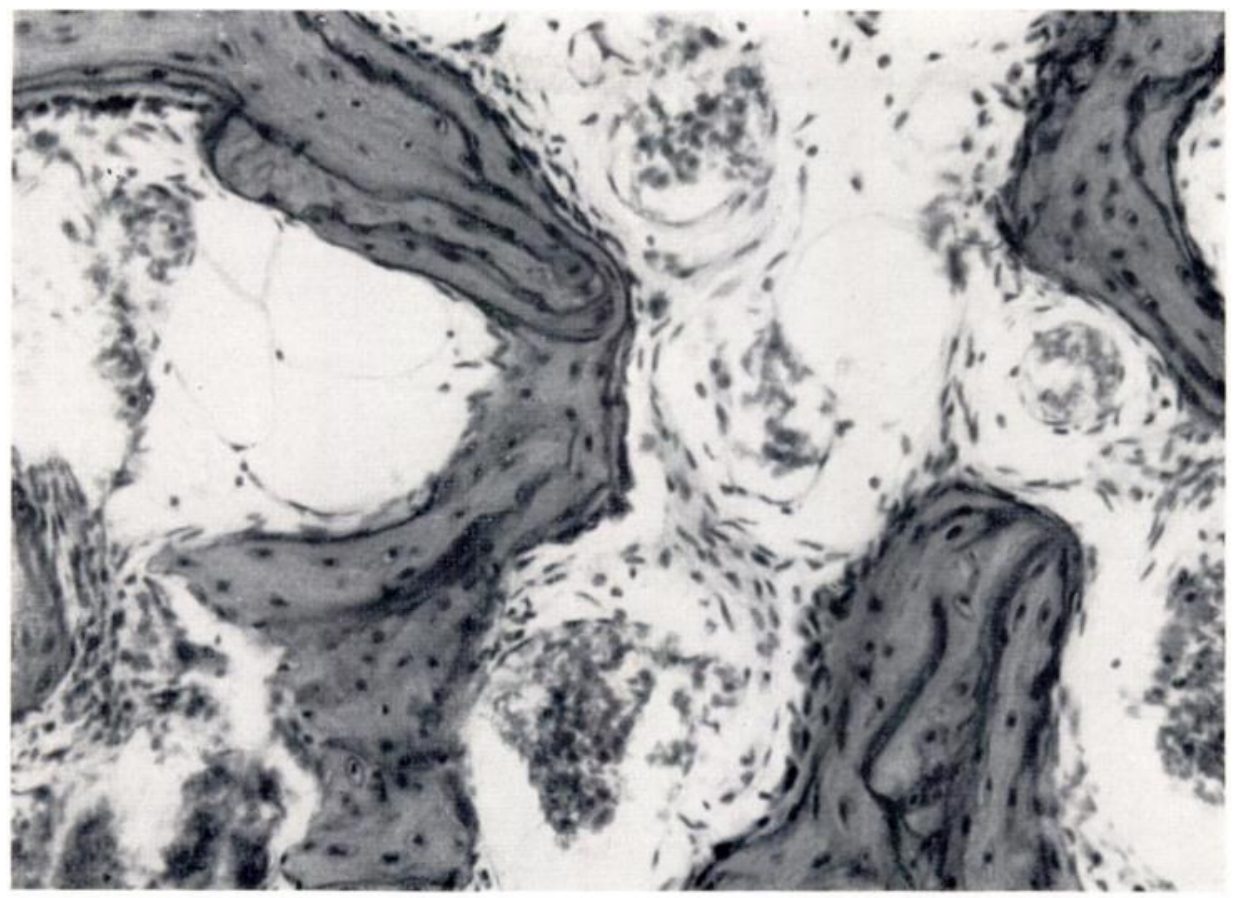

Fig. 9

The effect of four cycles of cortisone administration on the structure of the bone trabeculae. Repeated waves of resorption and deposition have left a series of irregular reversal lines in the bone structure. The marrow spaces are filled with greatly dilated vessels, a few fat cells and young connective tissue. $(\times 240$.

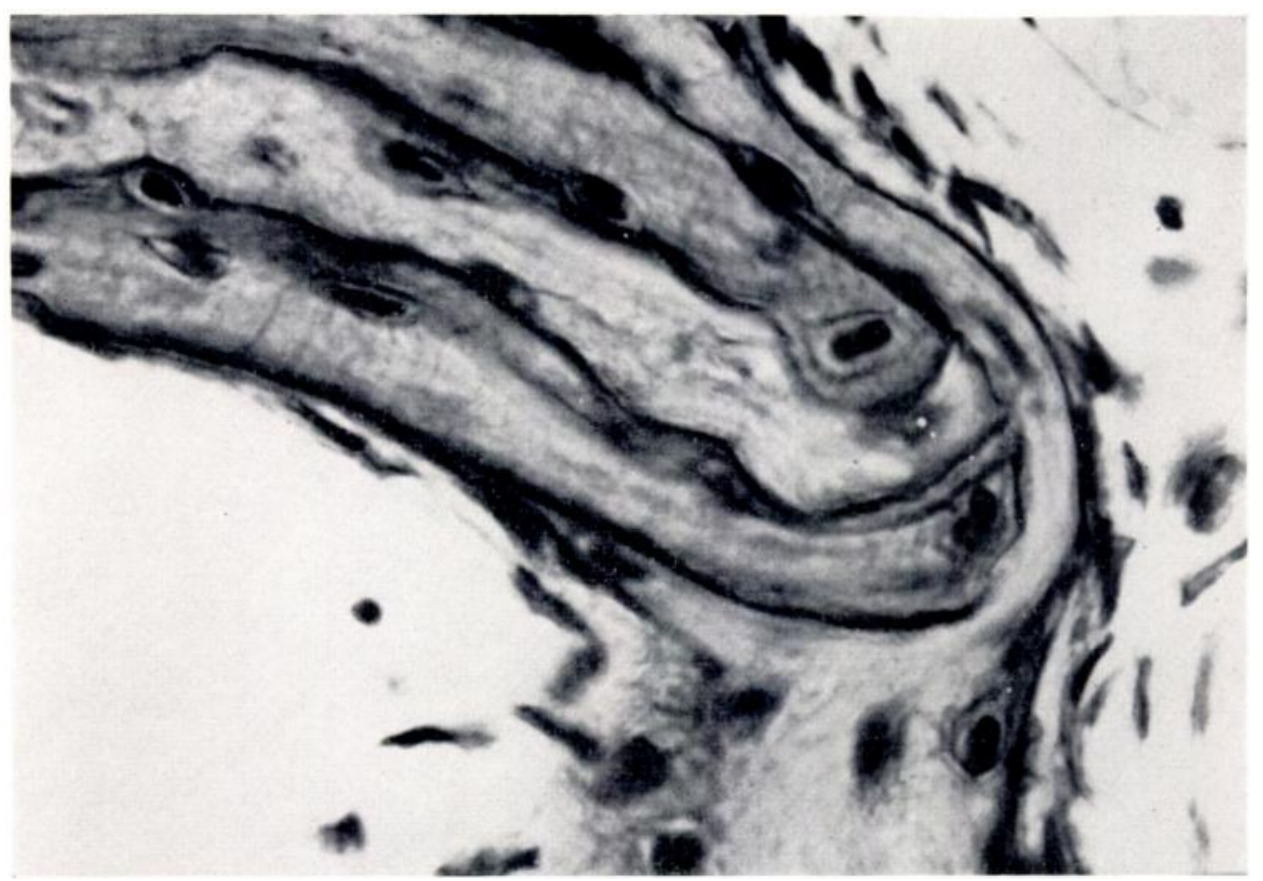

FIG. 10

Higher magnification of the section shown in Figure 9. A bone trabecula shows the increased number of new reversal lines and new bone formation. $(\times 1,000$.)

VOL. 40 B, NO. 1, FEBRUARY 1958 


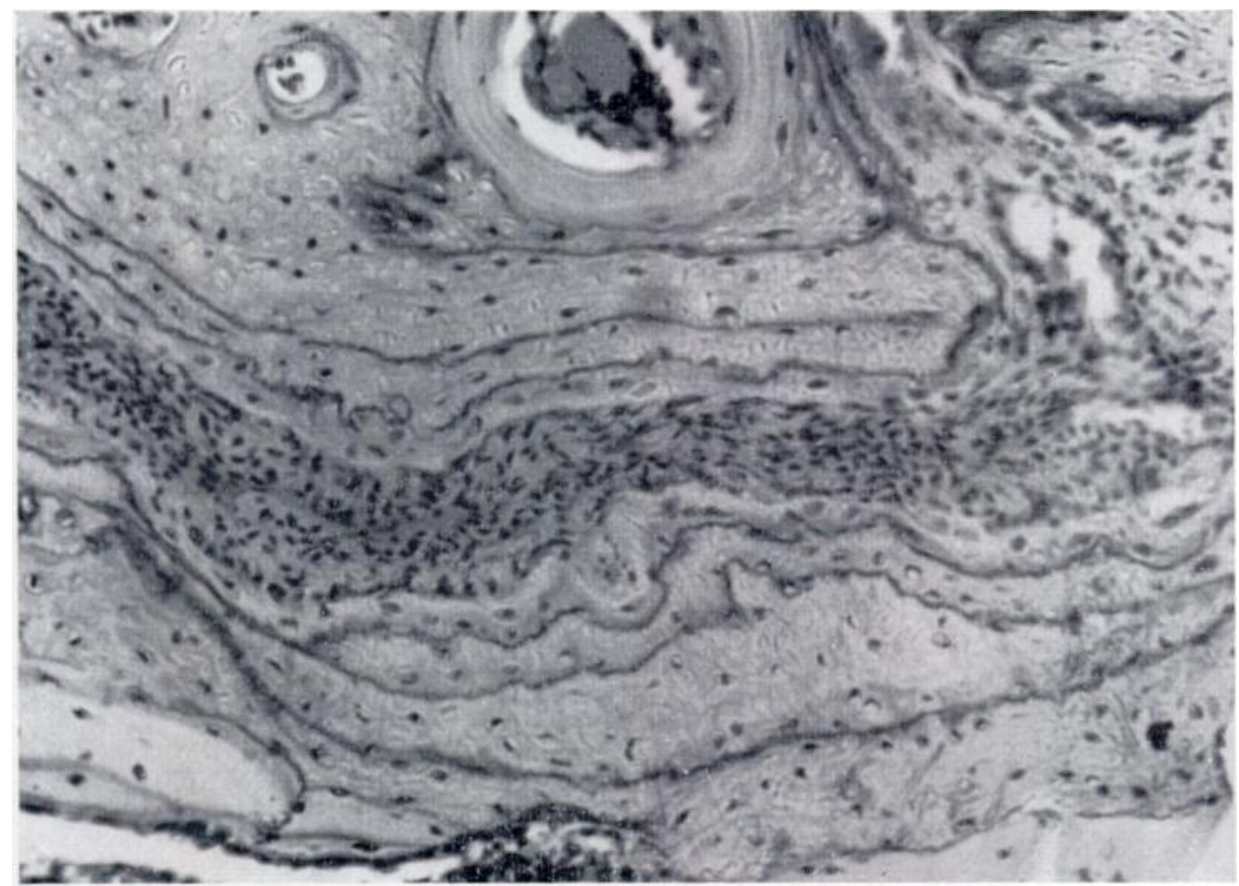

Fig. 11

The effect of three cycles of cortisone administration on the bone adjoining the sagittal suture. The wide layers of bone corresponding to fourteen days' withdrawal of cortisone are farthest from the sutural connective tissue. $(\times 240$. $)$

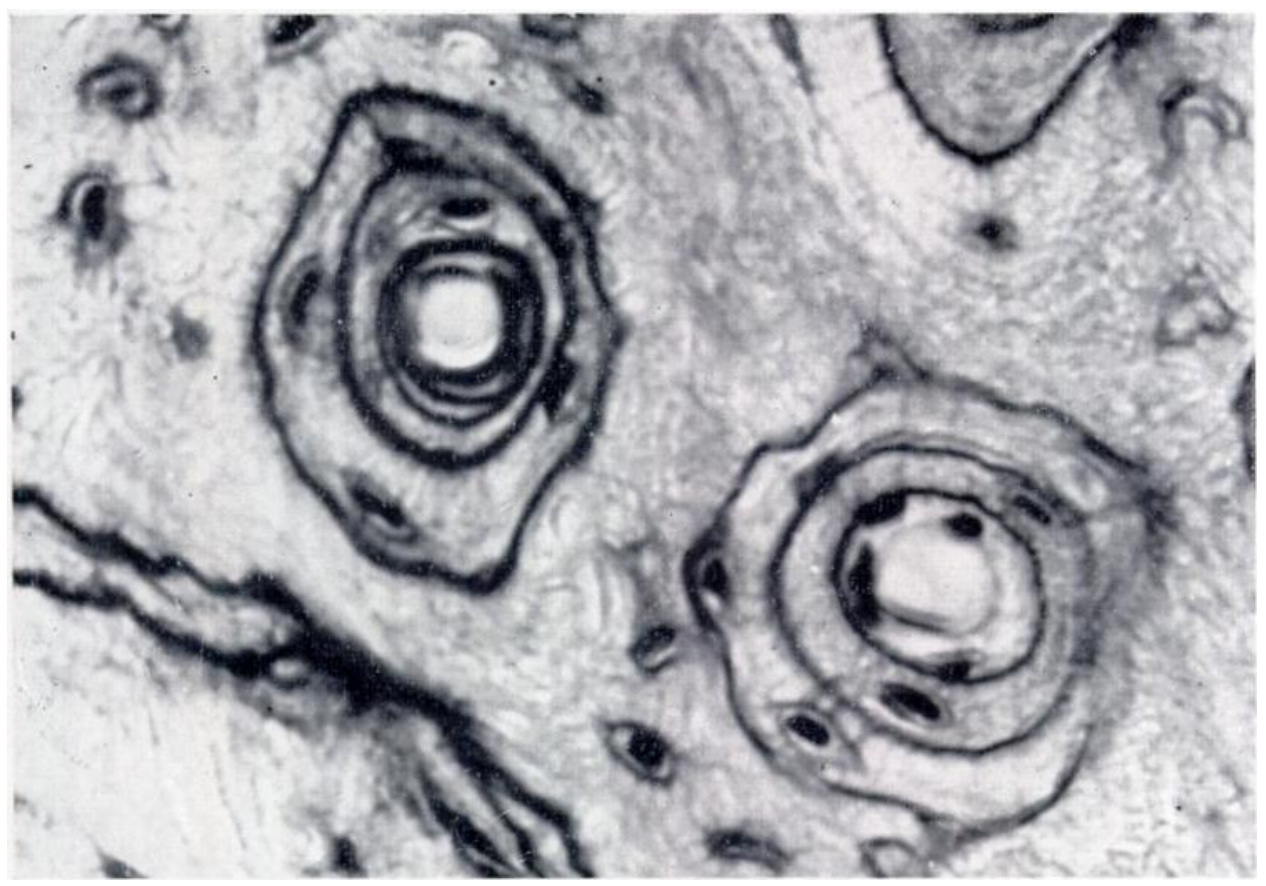

Fig. 12

The effect of four cycles of cortisone administration on the structure of Haversian systems: repeated "reversal" lines show where interruptions of bone formation occurred. In the lower left corner of the section three " reversal "lines converge to form a single thick line and then continue as two lines. $(\times 1,000$. 


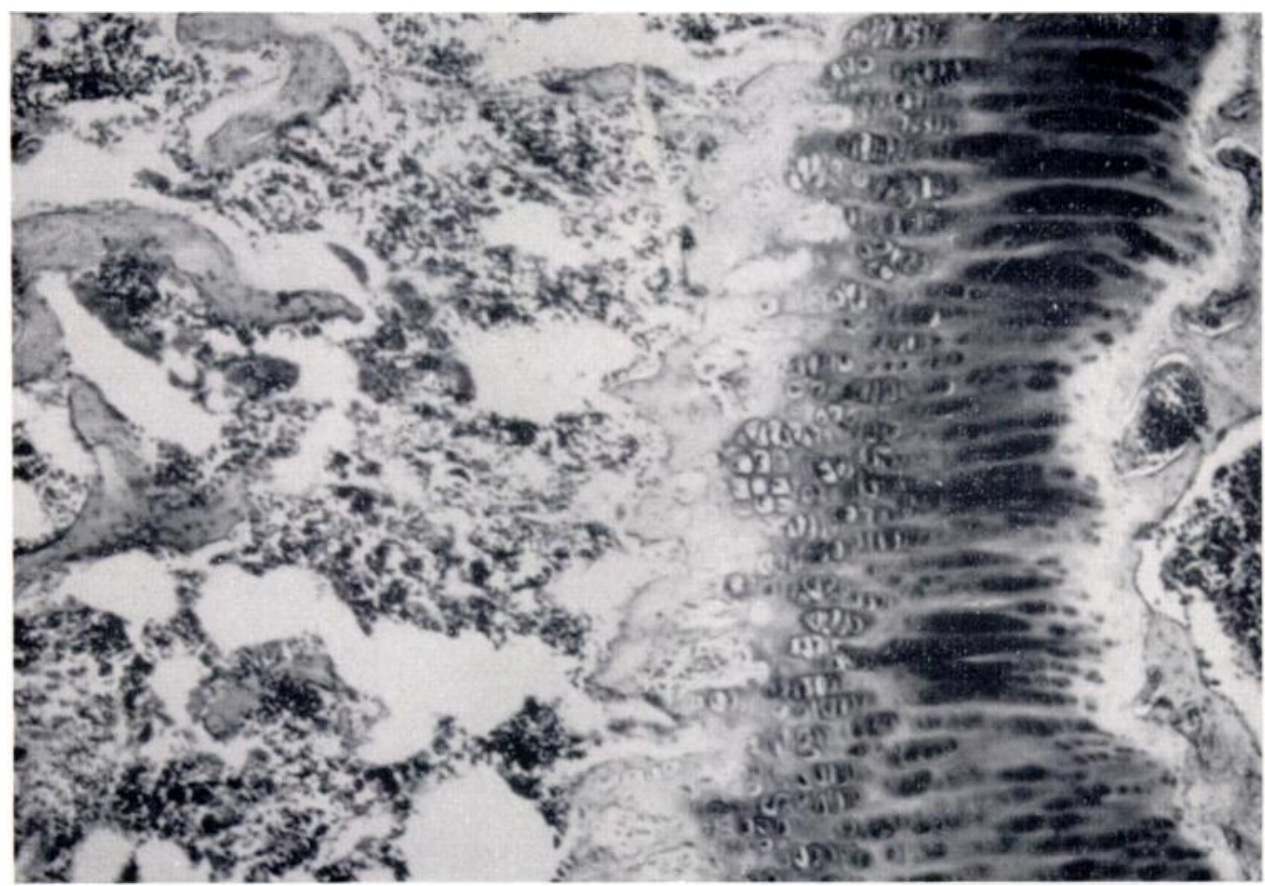

FIG. 13

The effect of four cycles of cortisone administration on the epiphysis of the lower end of the femur. The metaphysial cartilage is replaced by a thin layer of dense bone. $(\because 80$.

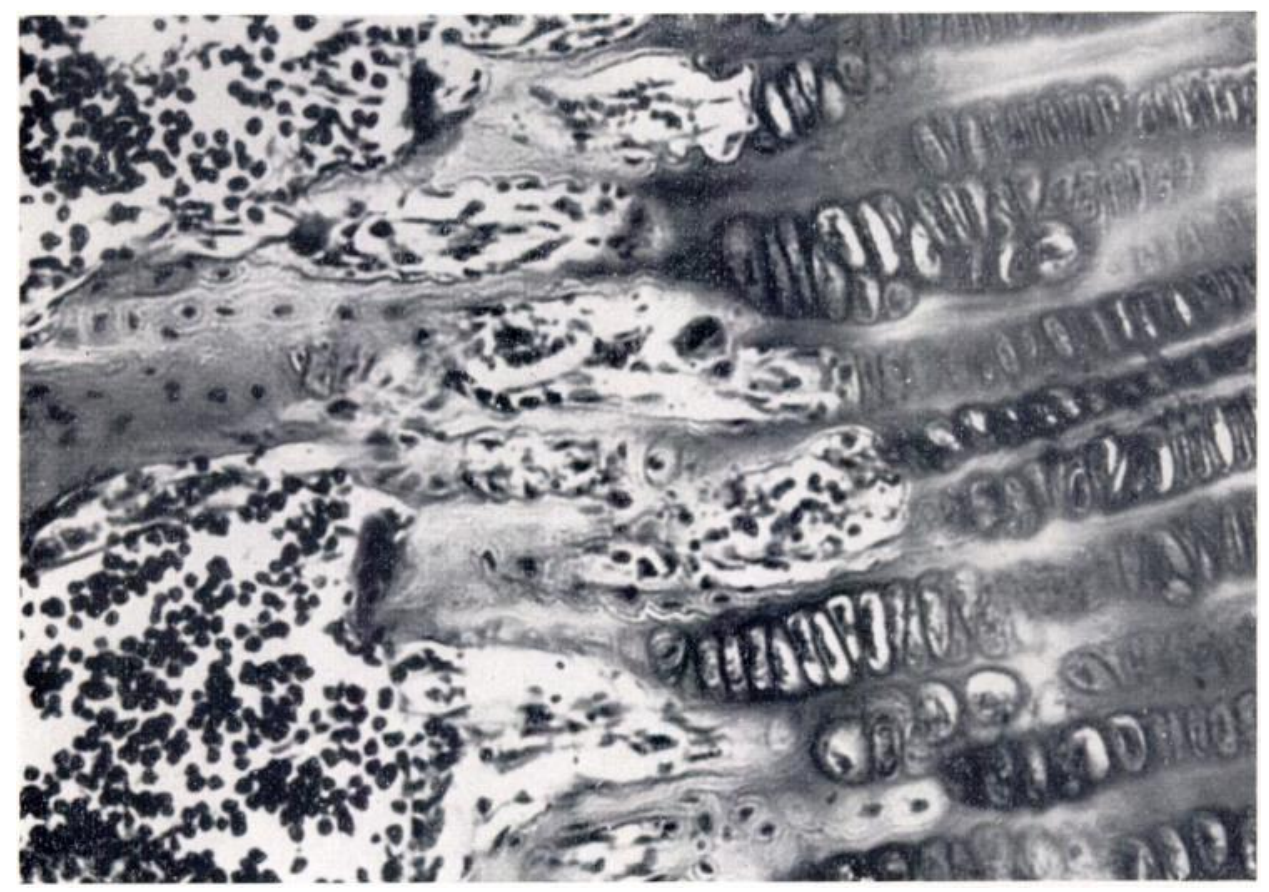

Fig. 14

Higher magnification of Figure 13. Formation of stunted bone trabeculae at the end of the irregularly arranged epiphysial cartilage cells. $(240$. 
Three cycles $(+7-14 ;+7-7 ;+7-7)-$ Three new layers of bone have formed on the outside and inside surfaces of the two cortical plates of the skull (Fig. 3). The new bone corresponding to the first cycle is two to three times as wide as the second and third layers. These layers of new bone are present also in the sagittal suture (Fig. 11), on the outer periosteal surface of the premaxilla, and towards the middle of the shaft of the femur. They show the same pattern; that is to say, the first layer formed is two to three times as wide as each of the second and third layers of bone.

The internal architecture of the bone is changing-around some vascular spaces there is a number of lines showing where repeated resorption and deposition have taken place.

Four cycles $(+7-7 ;+7-7 ;+7-14 ;+5-3)-\mathrm{A}$ young as well as an old animal were included in this experiment.

In the young animal four layers of new bone have formed on the outside and inside periosteal surfaces (Figs. 4 to 6) and in places within the sagittal and coronal sutures. The width of the new layers of bone varies according to the time for which cortisone had been withdrawn; the thickest layer is formed during fourteen days, the thinnest during three days. This last layer is not present in all sections; it is absent near the coronal suture and becomes prominent only towards the front of the cranial vault (Fig. 7). The thickness of the haematoxylin " reversal" line does not vary in any of these cycles, although the lines are much thicker and more darkly staining than those formed in the bone during the previous normal growth of the animal.

Similar new layers of bone have formed on the periosteal surfaces of the premaxilla and towards the centre of the shaft of the femur. These new layers of bone show the same distribution of width between the " reversal" lines.

The internal architecture of the bone, particularly in the skull, is beginning to change appreciably (Figs. 6 and 9). Some vascular spaces widen, showing increased vascularity and the presence of small osteoclasts at resorbing bone edges. Others show Haversian systems in which three or four concentric " reversal" lines are present around the central blood vessel (Fig. 12). Some trabeculae show an increased number of irregularly arranged "reversal" lines which correspond to the cycles of resorption and deposition (Fig. 10). The trabeculae are becoming irregularly arranged, and some of the marrow spaces are filled with extremely vascular connective tissue.

Significant changes have now taken place in the growing epiphysis of the femur (Figs. 13 and 14). There is a disappearance of long columns of metaphysial calcified cartilage; instead, a layer of new bone, which partly seals the epiphysial cartilage from the marrow space, is forming. The epiphysial cartilage is irregular in outline; cartilage cells vary greatly in development at the same level and some are enclosed in the newly formed bone.

The same pattern of new appositional bone growth seen in the young animal is shown in the old animal, but the changes are much less obvious (Fig. 8). Appositional bone growth has occurred in some places, but not over the whole cranial vault. The lines separating these layers of new bone either converge at points to form one or two lines within the bone, or disappear completely. Within the suture much more extensive (and possibly more rapid) changes have taken place. There are no lines indicating new layers of bone; instead there are large areas of haematoxylin-staining granules. Around small vessels some changes are found, but not to the extent shown in the young animal.

\section{DISCUSSION}

The intermittent administration of cortisone in the rabbit is associated with cycles of resorption and deposition of new bone in both growing and mature rabbits. In the young animal attention is directed to new growth of bone by darkly staining haematoxylin lines partitioning it into layers. These lines are more obvious and less regular than those formed 
previously in the bone. They are probably best designated as " reversal lines," because they form at the place where (and the time when) resorption is followed by deposition of bone. The width of the new layers of bone is of the same order, for the same time interval, in different animals of the same age, but in the old rabbit less bone forms. When different areas in the same animals are compared the amount of new bone varies-on the frontal aspect the line of bone is wider than on the occipital aspect of the skull, and relatively little forms in the middle of the shaft of the femur.

It is noteworthy that new layers of bone form not only on the outer, but also on the inner. aspect of the skull. The appearance of lines within the sagittal suture shows that separation of these bones must occur during growth of the skull. Indeed, the placing of lines within bone substance in this way could be used as an accurate indicator of the extent and direction of growth of skull bones.

Although the general emphasis is on active new bone formation after the withdrawal of cortisone, the influence of local factors modifies the response so that in some areas resorption of bone continues. Similarly, whilst appositional bone growth can be induced by these cycles of hormonal changes, new bone growth does not take place to the same extent in all bones. In some areas no bone forms, and in others reversal lines converge and run into one another to form single wide lines.

The internal architecture of the bone slowly changes with these repeated cycles of resorption and deposition of bone. This is particularly obvious round vessels within the bone, where waves of resorption followed by deposition leave the irregular deeply staining haemotoxylin lines in the bone substance. Bone trabeculae also show this-numerous " reversal" lines are present and there are even irregularities in the arrangement of trabeculae.

The appositional growth associated with the intermittent administration of cortisone may be due to periodic interruptions in the normal continuous growth of bones. It is known that cortisone inhibits the growth of bone in the rabbit (Sissons and Hadfield 1955) and since it is also known to influence the pituitary gland (Halmi and Barker 1952) the effect may be by way of change in the growth hormone. This may be true for young animals but, in the case of the older non-growing animal, this would not be so likely. Here it is probable that growth, after the withdrawal of cortisone, is in part due to a " rebound " phenomenon activating the endocrine systems concerned with growth. As the administration of cortisone is associated with atrophy of the adrenal cortex (Ingle and Mason 1938) its rapid withdrawal probably leads to a relative deficiency of cortical secretion. This temporarily induced "medical adrenalectomy " may be associated with the observed overgrowth of bone, as adrenalectomy in the well-fed rat is followed by an increased epiphysial growth (Wyman and Tum-Suden 1945) and accelerated regrowth of hair (Zeckwer 1953). Both of these observations are ascribed to increased secretion of growth hormone.

There are changes at the epiphyses of the growing bones following these cycles of resorption and deposition. A new layer of dense bone is forming which already partly seals off the epiphysial cartilage from the marrow space. This change differs from that seen after the administration of A.C.T.H. and cortisone in the rabbit (Storey 1957b).

It is noteworthy that at the time that cartilaginous growth is slowing down, thickening of the skull and long bones is continuing by an appositional process. These two changes, to some extent, are parallel to those normally seen during the growth and maturation of the limbs of the skeleton, where growth in length occurs first and is followed by thickening of bones (Silberberg and Silberberg 1941). It is perhaps relevant to ask whether the maturation of bone is due to changes not only in quantity and type of endocrine secretion but also to an alteration from a more or less continuous to an intermittent or cyclic production of hormones. Such alterations in rates of endocrine activity are already known to occur during the sexual development of girls (Nathanson, Towne and Aub 1941).

It is clear that a wide variation of bone changes can be produced by the use of cortisone

VOL. $40 \mathrm{~B}$, NO. 1, FEBRUARY 1958 
in the rabbit. The continuous administration of cortisone leads to a general osteoporosis of the skeleton, whereas intermittent administration is associated with appositional growth of the skull and changes in the pattern of the bone structure; these are characterised by an intense increase of vascularity and the appearance of darkly staining wide irregular " reversal " lines in the bone substance. It is possible that by altering the duration of these cycles variations of this change may be produced, ranging from rarefying to sclerosing conditions of bone. Also, since stress areas are particularly susceptible to continuous cortisone administration (Storey 1957a), the localisation of these changes to areas of bone under abnormal stress is to be expected. Experiments dealing with the long-range effects of such cycles of resorption and deposition of bone are now in progress and will be reported later.

These bone changes, associated with increase in the amount of cortical adrenal hormone, are specific and resemble changes seen in naturally occurring diseases. These diseases may well be due to such influences. It is not intended at this stage to convey, however, that they are necessarily due to changes in the adrenal structure or secretion. Indeed, they may be due to the aberrant action of other ductless glands; they may well be due to various combinations of ductless gland activity.

Such hormonal disturbances may be " primary," but more likely they are themselves caused by (or are associated with) other disturbances of the vascular or nervous system or by other factors. The important point is that basic observations of the type given here form an essential groundwork for the elucidation of the more complex changes of naturally occurring diseases, without necessarily giving any immediate clue to their doubtless motley and protean pathogenesis.

\section{SUMMARY}

The intermittent administration of cortisone in both the young and the mature rabbit is associated with appositional bone growth on the periosteal surfaces of the cranium, premaxilla and middle of the shaft of the femur; each new layer of bone is separated from the next by a darkly haematoxylin-staining " reversal" line. The internal architecture of the bone also changes in consequence of the repeated waves of resorption and deposition of bone round vascular spaces.

Cartilaginous growth at the epiphysis in the young rabbit is also affected. The long columns of metaphysial cartilage are replaced by a layer of new bone which partly seals the epiphysial cartilage from the marrow spaces.

Thanks are due to Messrs Merck Co. who generously supplied the cortisone acetate used in these experiments. This work was carried out under a grant from the National Health and Medical Research Council of Australia.

\section{REFERENCES}

Halmi, N. S., and Barker, S. B. (1952): Histo-physiological Effects of Cortisone on Rat Pituitary and Thyroid. Endocrinology, 51, 127.

INGLE, D. J., and MASON, H. L. (1938): Subcutaneous Administration of Cortin Compounds in Solid Form to the Rat. Proceedings of the Society for Experimental Biology and Medicine, 39, 154.

JAFFe, H. L. (1933): Paget's Disease of Bone. Archives of Pathology, 15, 83.

JAFFe, H. L., Bodansky, A., and Blair, J. E. (1932): The Influence of Age and of Duration of Treatment on the Production and Repair of Bone Lesions in Experimental Hyperparathyroidism. Journal of Experimental Medicine, 55, 139.

Nathanson, I. T., Towne, L. E., and Aub, J. C. (1941): Normal Excretion of Sex Hormones in Childhood. Endocrinology, 28, 851.

SELYE, H. (1932): On the Stimulation of New Bone Formation with Parathyroid Extract and Irradiated Ergosterol. Endocrinology, 16, 547.

Silberberg, M., and Silberberg, R. (1941): Effects of Hormones on the Skeleton of Mice, Guinea Pigs and Rats. Endocrinology, 29, 475.

Sissons, H. A. (1949): Intermittent Periosteal Activity. Nature, 163, 1,001. 
Sissons, H. A., and Hadfield, G. J. (1955): The Influence of Cortisone on the Structure and Growth of Bone. Journal of Anatomy, 89, 69.

Smith, R., and Storey, E. (1952): The Importance of Force in Orthodontics. The Design of Cuspid Retraction Springs. Australian Journal of Dentistry, 56, 291.

Storey, E. (1953): Bone Changes Associated with Tooth Movement. A Radiographic Study. Australian Journal of Dentistry, 57, 57.

Storey, E. (1954): Bone Changes Associated with Tooth Movement. The Influence of the Menstrual Cycle on the Rate of Tooth Movement. Australian Journal of Dentistry, 58, 80.

Storey, E. (1957a): The Influence of Cortisone and A.C.T.H. on Bone Subjected to Mechanical Stress (Tooth Movement). Submitted for publication.

Storey, E. (1957b): The Effect of Continuous Administration of Cortisone and Its Withdrawal on Bone. Australian and New Zealand Journal of Surgery, 27, 19.

Weinmann, J. P., and Sicher, H. (1955): Bone and Bones. Second edition. St Louis: The C. V. Mosby Company. Wyman, L. C., and Tum-Suden, C. (1945): The Effect of Adrenalectomy on the Epiphyseal Cartilage of the Rat. Endocrinology, 36, 340.

ZECKWER, I. T. (1953): The Accelerating Effect of Adrenalectomy on Regrowth of Hair in the Thyroidectomised Rat. Endocrinology, 52, 39.

VOl. $40 \mathrm{~B}$, NO. 1, fEBRUARY 1958 